\title{
Correction to: Einstein four-manifolds of three-nonnegative curvature operator
}

\section{Peng $W u^{1}$}

Received: 18 June 2020 / Accepted: 18 June 2020 / Published online: 16 December 2020

(c) Springer-Verlag GmbH Germany, part of Springer Nature 2020

\section{Correction to: \\ Mathematische Zeitschrift (2019) 293:1489-1511 \\ https://doi.org/10.1007/s00209-019-02296-8}

This is to acknowledge that the main results of the article [3] were first proved in a joint preprint with Xiaodong Cao [1]. Also, Corollary 1.2 had appeared earlier in [2, Theorem 1.1], which was proved there with "an adaptation of arguments in [1]," among others.

\section{References}

1. Cao, X., Wu, P.: Einstein four-manifolds of three-nonnegative curvature operator, preprint (2013). http://pi. math.cornell.edu/ cao/research.html. http://homepage.fudan.edu.cn/wupenguin/?page_id=116\&lang=en

2. Cao, X., Tran, H.: Einstein four-manifolds of pinched sectional curvature. Adv. Math. 335, 322-342 (2018)

3. Wu, P.: Einstein four-manifolds of three-nonnegative curvature operator. Math. Z. 293, 1489-1511 (2019)

Publisher's Note Springer Nature remains neutral with regard to jurisdictional claims in published maps and institutional affiliations.

The original article can be found online at https://doi.org/10.1007/s00209-019-02296-8.

$凶$ Peng Wu

wupenguin@fudan.edu.cn

1 Shanghai Center for Mathematical Sciences, Fudan University, Shanghai, China 